\title{
Acute exacerbation of paraneoplastic neurological syndrome after massive tumor lysis of neuroendocrine carcinoma by chemoradiotherapy
}

\author{
Yuki Yoshino $\cdot$ Shoko Akiyama $\cdot$ Kota Ouchi $\cdot$ Takayuki Oishi $\cdot$ \\ Hidekazu Takahashi · Jin Lee - Shin Takahashi • Hideki Shimodaira • \\ Shunsuke Kato • Chikashi Ishioka
}

Received: 21 December 2012/ Accepted: 17 March 2013/Published online: 4 April 2013

(C) The Japan Society of Clinical Oncology 2013

\begin{abstract}
We present the case of a 68-year-old woman who presented with a swelling on the left side of her lower jaw. Biopsy revealed neuroendocrine carcinoma of the mandible. Chemoradiotherapy with carboplatin and etoposide with local irradiation induced very rapid remission of the tumor. The massive tumor remission caused severe exacerbation of symptoms, including fatigue and muscle weakness. Her condition was diagnosed as paraneoplastic neurological syndrome with generalized peripheral nerve paralysis and respiratory failure. The syndrome was thought to be triggered by an accelerated immune reaction against the tumor, which attacked neurons with similar antigens to the tumor. The immune attack resulted in overt neurological disturbance that was not well recognized at the time of tumor diagnosis.
\end{abstract}

Keywords Paraneoplastic syndrome - Peripheral neuropathy $\cdot$ Tumor lysis $\cdot$ Neuroendocrine cancer

\section{Introduction}

Paraneoplastic neurological syndromes (PNS) are immunemediated neurological disorders triggered by immune

Y. Yoshino $\cdot$ K. Ouchi $\cdot$ T. Oishi $\cdot$ H. Takahashi $\cdot$ J. Lee

H. Shimodaira $\cdot$ S. Kato $\cdot$ C. Ishioka $(\bowtie)$

Department of Clinical Oncology, Institute of Development,

Aging, and Cancer, Tohoku University, Seiryo-machi 4-1,

Aoba-ku, Sendai 980-8575, Japan

e-mail: chikashi@idac.tohoku.ac.jp

S. Akiyama $\cdot$ S. Takahashi $\cdot$ C. Ishioka

Department of Clinical Oncology, Tohoku University

Hospital, Tohoku University, Seiryo-machi 1-1, Aoba-ku,

Sendai 980-8574, Japan responses to tumor cells. Some autoantibodies such as anti$\mathrm{Hu}$ or anti-Yo antibodies have well-defined relationships with paraneoplastic syndromes induced by neoplasia. However, a larger number of patients do not have such characterized antibodies or well-defined classical syndromes; instead, they have possible or gray zone clinical syndromes. We report the case of a 68 -year-old woman with possible PNS that rapidly deteriorated following massive tumor lysis induced by chemoradiotherapy.

\section{Case report}

A 68-year-old woman presented to the Tohoku University Hospital with a complaint of a swelling on the left side of her lower jaw. An otorhinolaryngologist performed a fineneedle biopsy (FNA), the findings of which led to the suspicion of primary malignant lymphoma. To confirm the diagnosis, an open biopsy of the primary tumor was performed. On the basis of histopathological and computed tomography (CT) findings, the patient was diagnosed with stage IV neuroendocrine carcinoma. She was then examined by medical oncologists at the hospital.

On examination, the tumor was $8.7 \times 7.1 \mathrm{~cm}$ in diameter, hard, slightly reddish, and with no tenderness (Fig. 1a). The European Cooperative Oncology Group performance status of the patient was grade 2 because of high-grade fatigue and mild muscle weakness (manual muscle test, or MMT 4). Sensory functions and reflexes were almost normal. Laboratory data showed no significant abnormality other than a neuron-specific enolase (NSE) level of $39 \mathrm{ng} / \mathrm{ml}$ (normal $<10 \mathrm{ng} / \mathrm{ml}$ ), a soluble interleukin 2 receptor level of $503 \mathrm{U} / \mathrm{ml}$ (normal $<496 \mathrm{U} / \mathrm{ml}$ ), and a glycated hemoglobin level of $6.8 \%$ (normal $<6.2 \%$ ). The pathological diagnosis was neuroendocrine carcinoma 


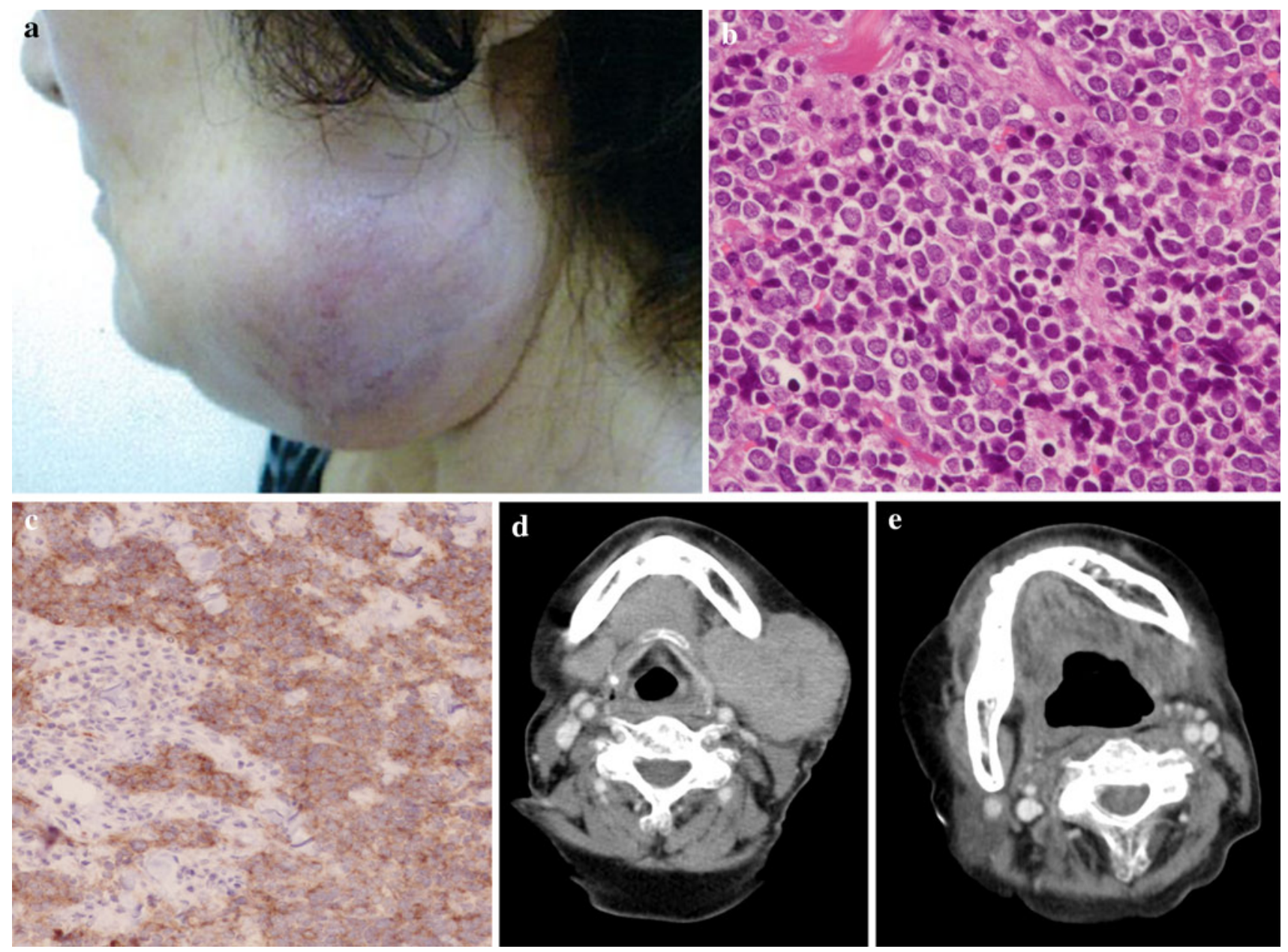

Fig. 1 The inspection of the tumor at the first examination, the histopathological findings, and the computed tomography (CT) images before and after chemoradiotherapy. a The submandibular tumor is large, slightly reddish, hard, and with no tenderness. b H\&E staining reveals densely clustered, small round cells with large nuclei, typical of neuroendocrine cancer. c Ki-67 staining of the tumor

(Fig. 1b) comprising tumor cells that were negative for chromogranin A and thyroid transcription factor- 1 and positive for synaptophysin, CD56, and NSE. The Ki-67 index was $70 \%$ (Fig. 1c), indicating that the pathological classification was grade 3 neuroendocrine carcinoma according to the World Health Organization classification [1]. CT performed at this point revealed a large primary tumor (Fig. 1d) and several cervical lymph node metastases, but no distant metastasis. The clinical staging was T3N2bM0, stage IV according to the TNM classification of the Union for International Cancer Control, 7th edition.

The patient was treated with concurrent chemoradiotherapy wherein carboplatin $(364 \mathrm{mg}$; AUC $=5)$ on day 1 and etoposide $\left(128 \mathrm{mg} ; 80 \mathrm{mg} / \mathrm{m}^{2}\right)$ on days $1-3$ were administered with local irradiation (total $60 \mathrm{~Gy} ; 2$ Gy in 30 fractions). The tumor softened over several days after therapy initiation, and this was followed by very rapid reveals approximately $70 \%$ positive tumor cells, indicating high growth capacity. d CT performed on initial presentation reveals a large tumor and several enlarged lymph nodes. e CT performed 2 months after the first chemoradiotherapy reveals a markedly shrunk primary tumor with no enlarged lymph nodes

shrinkage (Fig. 1e). However, general fatigue increased considerably, and overt respiratory failure with severe hypercapnia developed on day 8 after therapy initiation. Her respiratory and systemic muscle tones were severely weakened (MMT 0-1). Reflexes had almost disappeared. On the other hand, sensory functions were preserved. She was intubated, and forced mechanical ventilation was initiated. No severe abnormalities were found on laboratory tests.

We consulted with neurologists in the hospital to evaluate her symptoms. Neurophysiological examinations revealed widespread axonal damage of the peripheral nerves, which is similar to the severe subtype of GuillainBarré syndrome (GBS). No anti-Hu, anti-acetylcholine receptor, anti-GM1, or anti-GQ1b antibody was detected. We diagnosed her with paraneoplastic neurological syndrome. The chemoradiotherapy was terminated, and 
intravenous high-dose gamma globulin (IVIG, $15 \mathrm{~g} /$ day) and methylprednisolone pulse $(1,000 \mathrm{mg} /$ day $)$ therapy were administered for 5 and 3 days, respectively. Respiratory paralysis improved slightly for a few weeks, and forced mechanical ventilation was weaned to supported spontaneous respiration. Her consciousness was clear after sedation was stopped, although she was unable to speak because of a tracheotomy. Meanwhile, the tumor continued to regress, and several weeks later, it almost disappeared as was evident by inspection, palpation, and ultrasonography. The severe adverse effects of the chemoradiotherapy were grade 4 leukopenia (leukocyte count $600 / \mu \mathrm{l}$ ), grade 4 neutropenia (neutrophil count $270 / \mu \mathrm{l}$ ), and grade 3 thrombocytopenia (platelet count $40,000 / \mu \mathrm{l}$ ) according to the Common Terminology Criteria for Adverse Events (CTCAE), version 4.0. No severe non-hematological adverse effects were observed.

A month after the onset of respiratory failure, the second course of IVIG was administered. Paralysis improved very slowly. Two weeks after the second IVIG, inspection revealed tumor regrowth. The same initial chemotherapy was reintroduced, and the tumor shrank after 2 courses. During the therapy, however, she suddenly lost consciousness. Brain CT revealed multiple cerebral infarctions. Unfortunately, we could not continue aggressive chemotherapy to achieve cancer remission, and palliative care was introduced. Because of this event, it is hard to assess whether the paralysis got worse after the second chemotherapy.

\section{Discussion}

PNS are classically thought to be quite rare, excluding Lambert-Eaton myasthenic syndrome, which arises in $3 \%$ cases of small cell lung cancers, and myasthenia gravis, which arises in $15 \%$ cases of thymic tumors [2]. Although such classical syndromes are rare, some degree of neurological disturbance or myopathy is relatively common in cancer patients and may be observed in approximately $50 \%$ patients $[3,4]$. Extensive exploration of neurological disorders in cancer patients can identify PNSs that are not well characterized.

Diagnosis of PNS requires exclusion of other causes of neuropathy. In this patient, most abnormalities were observed in motor functions. On the other hand, virtually no sensory disturbance was observed. This is incompatible with diabetic neuropathy or drug-induced neuropathy. Rather, immune-mediated neuropathy, such as GBS or PNS, is more plausible. Although there are no definitive clinical diagnostic criteria for PNS, some useful ones have been proposed. For example, Graus et al. [5] proposed criteria in which definite and possible PNSs are defined. Patients who meet one of their following four criteria are diagnosed with definite PNS: a classical syndrome and cancer that develops within 5 years of diagnosis of the syndrome; a nonclassical syndrome that improves after cancer treatment; a nonclassical syndrome with antibodies related to paraneoplastic syndromes and cancer that develops within 5 years of the diagnosis of the syndrome; and a neurological syndrome with well-characterized antibodies (anti-Hu, Yo, CV2, Ri, Ma2, or amphiphysin) and no cancer.

Besides the above, patients who meet one of the following three criteria are diagnosed with possible PNS: a classical syndrome, no cancer, but high risk of an underlying tumor; a neurological syndrome with partially characterized antibodies; and a nonclassical syndrome and cancer presenting within 2 years of diagnosis. According to these criteria, we diagnosed our patient with possible PNS.

Cellular and humoral autoimmune reactions against tumors that cross-react against neuronal components are thought to be important in the pathogenesis of PNS. Immune-modulating treatments such as plasma exchange, IVIG, and steroid pulse are used for PNS [6]. Because the blood pressure had not been stable in this patient, we used only IVIG and steroid pulse. To improve PNS definitely, removal of the antigen or the tumor cells is necessary. However, this is very difficult because such PNSs often occur in patients with advanced cancer that cannot be completely remitted by surgery or chemoradiotherapy. If PNS is identified with early stage cancer or cancer with high chemo- or radiosensitivity, such as hematological malignancy or neuroendocrine carcinoma, aggressive treatment may improve the syndromes [6]. Combination chemotherapy such as cisplatin and irinotecan, cisplatin and etoposide, and carboplatin and etoposide are used for neuroendocrine carcinoma [7]. For locoregional disease, concurrent radiotherapy is also utilized [7]. In this patient, we selected the concurrent chemoradiotherapy wherein carboplatin and etoposide were administered with local irradiation because of the limited disease extension, the patient's age, and the relatively low PS.

Some reports have shown that cytotoxic drugs, especially vinca alkaloids or oxaliplatin, or the tumor itself can induce neurological syndromes such as GBS over a period of weeks or longer [8-11]. Severe neurological disturbance in our patient appeared shortly after tumor lysis induced by chemoradiotherapy initiation; this disturbance was inconsistent with GBS induced by drug or tumor. Rather, the massive tumor lysis may have stimulated autoimmune reactions resulting in rapid destruction of neurons having similar antigens to the tumor. The mild muscle weakness and fatigue before the introduction of the first chemoradiotherapy are more compatible with this hypothesis. To our knowledge, this is the first report that indicates a possible relationship between acute exacerbation of PNS and 
tumor lysis. Unfortunately, an unpredictable event, cerebral infarction, occurred during the second chemotherapy, necessitating immediate discontinuation of aggressive treatment. Therefore, we could not show whether or not her paralysis improved after tumor remission.

For patients who suffer from paraneoplastic syndrome, treatment of the tumor is essential to improve the syndrome. However, PNSs are often diagnosed when neurological symptoms are alleviated after tumor remission. In addition, rapid tumor regression may exacerbate PNS, as seen in the present patient. This case report showed that undetected PNS may suddenly appear when intensive cytotoxic therapy causes tumor lysis. Because of these dilemmas, more definitive criteria for PNS diagnosis are urgently required to develop reasonable treatment strategies.

Acknowledgments The authors would like to thank Dr. Fumiyoshi Fujishima and Dr. Mika Watanabe for taking pictures of the pathological images and Enago (http://www.enago.jp) for the English language review.

Conflict of interest The authors have no conflict of interest to disclose with respect to this manuscript.

\section{References}

1. Klimstra DS, Modlin IR, Coppola D et al (2010) The pathologic classification of neuroendocrine tumors: a review of nomenclature, grading, and staging systems. Pancreas 39:707-712
2. Rudnicki SA, Dalmau J (2000) Paraneoplastic syndromes of the spinal cord, nerve, and muscle. Muscle Nerve 23:1800-1818

3. Paul T, Katiyar BC, Misra S et al (1978) Carcinomatous neuromuscular syndromes. A clinical and quantitative electrophysiological study. Brain 101:53-63

4. Trojaborg W, Frantzen E, Andersen T (1969) Peripheral neuropathy and myopathy associated with carcinoma of the lung. Brain 92:71-82

5. Graus F, Delattre JY, Antoine JC et al (2004) Recommended diagnostic criteria for paraneoplastic neurological syndromes. J Neurol Neurosurg Psychiatry 75:1135-1140

6. Pelosof LC, Gerber DE (2010) Paraneoplastic syndromes: an approach to diagnosis and treatment. Mayo Clin Proc 85:838-854

7. Strosberg JR, Coppola D, Klimstra DS et al (2010) The NANETS consensus guidelines for the diagnosis and management of poorly differentiated (high-grade) extrapulmonary neuroendocrine carcinomas. Pancreas 39:799-800

8. Johnson NT, Crawford SW, Sargur M (1987) Acute acquired demyelinating polyneuropathy with respiratory failure following high-dose systemic cytosine arabinoside and marrow transplantation. Bone Marrow Transplant 2:203-207

9. Moudgil SS, Riggs JE (2000) Fulminant peripheral neuropathy with severe quadriparesis associated with vincristine therapy. Ann Pharmacother 34:1136-1138

10. Sarmiento MA, Neme D, Fornari MC et al (2000) Guillain-Barre syndrome following 2-chlorodeoxyadenosine treatment for Hairy Cell Leukemia. Leuk Lymphoma 39:657-659

11. Yoon JY, Nam TS, Kim MK et al (2012) Acute inflammatory demyelinating polyradiculoneuropathy in a patient receiving oxaliplatin-based chemotherapy. Asia Pac J Clin Oncol 8: 201-204 